\title{
Subclinical Infection of Macaques and Baboons with A Baboon Simarterivirus
}

\author{
Connor R. Buechler 1,2, Matthew Semler ${ }^{1,2}$, David A. Baker ${ }^{1,2}$, Christina Newman 1,2, \\ Joseph P. Cornish ${ }^{3}$, Deborah Chavez ${ }^{4}$, Bernadette Guerra ${ }^{4}$, Robert Lanford ${ }^{4}$, Kathy Brasky ${ }^{4}$, \\ Jens H. Kuhn ${ }^{5}\left(\mathbb{D}\right.$, Reed F. Johnson ${ }^{3}$, David H. O'Connor ${ }^{1,2}$ and Adam L. Bailey ${ }^{1,2,6, * \mathbb{D}}$ \\ 1 Department of Pathology and Laboratory Medicine, University of Wisconsin-Madison, Madison, WI 53711, \\ USA; connor.buechler@gmail.com (C.B.); msemler1656@gmail.com (M.S.); dabaker3@wisc.edu (D.A.B.); \\ cnewman7986@gmail.com (C.N.); dhoconno@wisc.edu (D.H.O.) \\ 2 Wisconsin National Primate Research Center, Madison, WI 53711, USA. \\ 3 Emerging Viral Pathogens Section, Laboratory of Immunoregulation, Division of Intramural Research, \\ National Institute of Allergy and Infectious Diseases, National Institutes of Health, Frederick, MD 20896, \\ USA; joseph.cornish@nih.gov (J.P.C.); johnsonreed@niaid.nih.gov (R.F.J.) \\ 4 Texas Biomedical Research Institute, Southwest National Primate Research Center, San Antonio, TX 78227, \\ USA; dchavez@txbiomed.org (D.C.); bguerra@txbiomed.org (B.G.); rlanford@txbiomed.org (R.L.); \\ kbrasky@txbiomed.org (K.B.) \\ 5 Integrated Research Facility at Fort Detrick, National Institute of Allergy and Infectious Diseases, \\ National Institutes of Health, Fort Detrick, Frederick, MD 21702, USA; kuhnjens@niaid.nih.gov \\ 6 Department of Pathology and Immunology, Washington University in St. Louis School of Medicine, \\ St. Louis, MO 63110, USA \\ * Correspondence: adam.bailey@wustl.edu; Tel.: +1-314-723-4604
}

Received: 8 November 2018; Accepted: 6 December 2018; Published: 10 December 2018

\begin{abstract}
Simarteriviruses (Arteriviridae: Simarterivirinae) are commonly found at high titers in the blood of African monkeys but do not cause overt disease in these hosts. In contrast, simarteriviruses cause severe disease in Asian macaques upon accidental or experimental transmission. Here, we sought to better understand the host-dependent drivers of simarterivirus pathogenesis by infecting olive baboons $(n=4)$ and rhesus monkeys $(n=4)$ with the simarterivirus Southwest baboon virus 1 (SWBV-1). Surprisingly, none of the animals in our study showed signs of disease following SWBV-1 inoculation. Three animals (two rhesus monkeys and one olive baboon) became infected and sustained high levels of SWBV-1 viremia for the duration of the study. The course of SWBV-1 infection was highly predictable: plasma viremia peaked between $1 \times 10^{7}$ and $1 \times 10^{8}$ vRNA copies $/ \mathrm{mL}$ at 3-10 days post-inoculation, which was followed by a relative nadir and then establishment of a stable set-point between $1 \times 10^{6}$ and $1 \times 10^{7}$ vRNA copies $/ \mathrm{mL}$ for the remainder of the study (56 days). We characterized cellular and antibody responses to SWBV-1 infection in these animals, demonstrating that macaques and baboons mount similar responses to SWBV-1 infection, yet these responses are ineffective at clearing SWBV-1 infection. SWBV-1 sequencing revealed the accumulation of non-synonymous mutations in a region of the genome that corresponds to an immunodominant epitope in the simarterivirus major envelope glycoprotein GP5, which likely contribute to viral persistence by enabling escape from host antibodies.
\end{abstract}

Keywords: Arteriviridae; simarterivirus; SHFV; simian hemorrhagic fever virus; southwest baboon virus 1; SWBV-1

\section{Introduction}

Wild non-human primates are an important reservoir of several zoonotic pathogens, and recent surveys have shown that many harbor microbes with unknown zoonotic potential [1-4]. In particular, 
a high proportion of wild monkeys across sub-Saharan Africa harbor arteriviruses (order: Nidovirales; family: Arteriviridae) that have a unique genomic architecture (subfamily: Simarterivirinae) $[5,6]$. In naturally-infected wild African monkeys, simarteriviruses cause persistent high-titer viremia and exhibit a high degree of intra- and inter-host genetic variation without eliciting any overt signs of disease-features that may make cross-species transmission to humans more likely [5]. However, the course of simarterivirus infection in African monkeys, including the acute-phase of infection, is not well characterized.

Wild Asian macaques (Macaca spp.) are not known to harbor simarteriviruses. However, captive macaques have been infected with simarteriviruses (both experimentally and unintentionally) and develop severe and frequently fatal disease characterized by hemorrhage and multi-organ failure, with lethality often exceeding 50\% [7-18]. Indeed, the first two simarteriviruses identified, simian hemorrhagic encephalitis virus (SHEV) and simian hemorrhagic fever virus (SHFV), were first encountered in 1964 following two seemingly unconnected outbreaks of viral hemorrhagic fever in captive macaques at primate facilities in Sukhumi, Georgian SSR, USSR, and the National Institutes of Health in Bethesda, Maryland, USA, respectively [7]. Since the discovery of these two viruses, many other outbreaks have occurred in captive Asian macaques throughout the world, with captive co-housed African monkeys often implicated as the source of infection. Recent retrospective investigations indicate that many of these outbreaks may have been caused by viruses distinct from SHEV and SHFV, suggesting that simarteriviruses as a group may be universally pathogenic to Asian macaques [19]. Of note, not all simarteriviruses are universally fatal in macaques. Crab-eating macaques (Macaca fascicularis) infected with the simarterivirus Kibale red colobus virus 1 (KRCV-1) isolated from wild red colobus (Procolobus rufomitratus tephrosceles) in Uganda exhibited mild signs of disease and fully recovered upon clearance of KRCV-1 viremia [18].

We recently discovered a novel simarterivirus circulating in apparently healthy olive baboons (Papio anubis) at the Southwest National Primate Research Center (SNPRC) in San Antonio, Texas, USA, that we termed Southwest baboon virus (SWBV-1, hereafter referred to as "SWBV") [20]. Using this virus, we sought to understand differences in simarterivirus infection and disease in natural (i.e., baboon) versus non-natural (i.e., macaque) hosts that are $98 \%$ genetically identical. In this study, we have the broader goal of understanding host factors that facilitate or protect against the development of viral hemorrhagic fever. Concurrently, a similar study was conducted in patas monkeys (Erythrocebus patas) and rhesus monkeys (Macaca mulatta) using SHFV (see article by Cornish et al., currently under review).

\section{Materials and Methods}

\subsection{Study Design}

The experimental design and protocols were agreed upon prior to initiation of the study and were not modified after the study had begun. Animal care, observations, and clinical laboratory testing were performed at the Southwest National Primate Research Center (SNPRC) in San Antonio, Texas, USA. Viral loads, flow cytometry, and data analysis were performed in batch at the Wisconsin National Primate Research Center (WNPRC), Madison, Wisconsin, USA, after conclusion of the study.

\subsection{Care and Ethical Use of Animals}

All baboons and macaques used in this study were cared for by the staff at the Southwest National Primate Research Center in accordance with the regulations and guidelines outlined in the Animal Welfare Act and the Guide for the Care and Use of Laboratory Animals. Details of this study (protocol 1539 PC, MM 0) were approved by the Texas Biomedical Research Institute's Animal Care and Use Committee in November of 2016, in accordance with recommendations of the Weatherall Report. 


\subsection{Clinical Scoring}

Animals were observed for signs of disease and scored according to the parameters described below. Scores equal to or greater than 5 required evaluation by the study veterinarian. Scores equal to or greater than 15 were deemed terminally ill. Weight loss: $0=$ decrease of $0-10 \%$; $1=$ decrease of $10-20 \% ; 2=$ decrease of $\geq 20 \%$. Temperature: $0=$ change $<2{ }^{\circ} \mathrm{F} ; 1=$ change of $2-3{ }^{\circ} \mathrm{F}$; $2=$ change of $3-4{ }^{\circ} \mathrm{F} ; 3=$ change of $\geq 5^{\circ} \mathrm{F}$. Responsiveness: $0=$ alert, responsive, normal activity, free of disease signs or exhibits only resolved/resolving disease signs; 1 = slightly diminished general activity, subdued but responds normally to external stimuli; $2=$ withdrawn, may have head down, fetal posture, hunched, reduced response to external stimuli; $8=$ prostrate but able to rise if stimulated, moderate to dramatically reduced response to external stimuli; $15=$ persistently prostrate, severely or completely unresponsive, may have signs of respiratory distress. Hair coat: $0=$ normal appearance; $1=$ rough hair coat. Respiration: $0=$ normal breathing; $3=$ labored; $15=$ agonal. Petechia: $0=$ none; $1=$ mild $(1-39 \%) ; 2=$ moderate $(40-79 \%) ; 3=$ severe $(\geq 80 \%)$. Bleeding: $0=$ none; $1=$ at bleeding site; $2=$ other than bleeding site. Nasal discharge: $0=$ not present; $1=$ present. Chow eaten: $0=25-100 \% ; 1=<25 \%$. Food enrichment: $0=25-100 \% ; 1=<25 \%$. Stool: $0=$ normal; $1=$ no stool present; $2=$ diarrhea. Fluid Intake: $0=$ drinking normal amounts; $1=$ reduced fluid intake; $2=$ no intake. Skin tenting: $0=$ normal, $1=\geq 2 \mathrm{~s}$.

\subsection{Virus Inoculations}

A SWBV stock was created for this study by aliquoting plasma collected from olive baboon 15290 , which was found to be viremic with SWBV via RT-qPCR during a screen of 30 baboons at SNPRC. Macaques and baboons infected with SWBV in this study were inoculated intramuscularly with 500 $\mu \mathrm{L}$ of plasma from this animal containing $1.7 \times 10^{5} \mathrm{vRNA}$ copies of SWBV.

\subsection{Deep Sequencing}

Samples were processed for sequencing in a biosafety level 3 laboratory. For each animal, viral RNA was isolated from approximately $200 \mu \mathrm{L}$ of plasma using the Qiagen QIAamp MinElute virus spin kit (Qiagen, Hilden, Germany), omitting carrier RNA. cDNA synthesis and PCR were performed using random hexamers (for the inoculum) or with seven primer sets (for the remaining animals, see Table S2) to amplify the entire SWBV genome in overlapping amplicons with the Superscript III One-Step RT-PCR kit with Platinum Taq Polymerase (Invitrogen, Carlsbad, CA, USA). All amplicons from a single sample were pooled to achieve $1 \mathrm{ng}$ of total amplified DNA in $5 \mu \mathrm{L}$. Pooled amplicons (or total cDNA generated from random-hexamer amplification) were fragmented, and sequencing adaptors were added using the Nextera XT DNA Library Preparation Kit (Illumina, San Diego, CA, USA). Indexed sequencing libraries were cleaned using AMPure XP beads (Agencourt Bioscience Corporation, Beverly, MA, USA) and quantified using the Qubit dsDNA HS Assay Kit with the Qubit fluorometer (Invitrogen, Carlsbad, CA, USA). Product length was measured using the Agilent 2100 Bioanalyzer High Sensitivity DNA kit (Life Technologies, Madison, WI, USA). Deep sequencing was performed on the Illumina MiSeq. Sequencing of SWBV from baboon 15290 was performed in 2016; all other sequencing was performed on the same MiSeq run in 2018. SWBV sequencing reads pertaining to this study can be found online [21].

\subsection{Sequence Read Mapping and Variant Calling}

Raw sequencing reads from baboon 15290 (the inoculum) were mapped to the previously-published SWBV-1 sequence (GenBank \#KM110947) to generate an inoculum consensus sequence (GenBank \#MH686150). Annotations were then transferred from KM110947 to MH686150 and checked manually. For baboon 31459 (12 days post-inoculation (DPI)), macaque 31721 (56 DPI), and macaque 31816 (56 DPI), reads were mapped to each of the seven amplicons individually. Ten thousand mapped reads were randomly subsampled from each amplicon in each sample, normalizing 
coverage at approximately $1000 \times$ per nucleotide site throughout the complete genome. The normalized reads were then mapped to the consensus sequence of the inoculum using the bbmap read mapper [22]. Minor variants that comprise at least $5 \%$ of total sequences in any of the four samples were identified using the bbmap callvariants.sh tool. The predicted effect of these variants on SWBV was calculated using snpEff [23] (Table S1). These sequencing datasets and Jupyter notebooks that create the data analysis environment and reproducibly perform the data analyses can be downloaded [24].

\subsection{Viral Loads}

A TaqMan RT-qPCR assay was developed to quantify SWBV-1 plasma viral RNA (forward primer: 5'-GCTTGCTGGTAAGATTGCCA-3'; reverse primer: 5'-GCAGCGGATCTTTGTGGAA-3'; probe: 5'-6FAM-TGATTAACCTGAGGAAGTATGGCTGGC-BHQ1-3'), as described in detail previously [20]. Briefly, RNA was extracted from $100 \mu \mathrm{L}$ of plasma using the Viral Total Nucleic Acid Purification Kit (Promega, Madison, WI, USA) on a Maxwell 16 MDx instrument and eluted in $50 \mu \mathrm{L}$. Reverse transcription and PCR were performed using the SuperScript III One-Step qRT-PCR system (Invitrogen, Carlsbad, CA, USA) on a LightCycler 480 (Roche, Indianapolis, IN, USA). Reverse transcription was carried out at $37{ }^{\circ} \mathrm{C}$ for $15 \mathrm{~min}$ and then $50{ }^{\circ} \mathrm{C}$ for $30 \mathrm{~min}$ followed by $2 \mathrm{~min}$ at $95^{\circ} \mathrm{C}$ and then 50 cycles of amplification as follows: $95^{\circ} \mathrm{C}$ for $15 \mathrm{~s}$ and $60{ }^{\circ} \mathrm{C}$ for $1 \mathrm{~min}$. The $50 \mu \mathrm{L}$-reaction mixture contained $5 \mu \mathrm{L}$ of extracted $\mathrm{RNA}, \mathrm{MgSO}_{4}$ at a final concentration of $3.0 \mathrm{mM}$, with the two amplification primers at a concentration of $500 \mathrm{nM}$ and probe at a concentration of $100 \mathrm{nM}$. RNA copy numbers were calculated using a standard curve (described previously in [20]) that was sensitive down to 10 copies of RNA transcript per reaction.

\subsection{Peptide Array}

Viral protein sequences were selected and submitted to Roche Sequencing Solutions (Madison, WI, USA) for development into a peptide microarray as part of an early access program. Protein sequences were predicted from the SWBV inoculum nucleotide sequence, and 16-mer peptides tiling each protein were designed to span each viral protein with a step size of 1 (amino acid overlap of 15). The peptide sequences were synthesized in situ with a Roche Sequencing Solutions Maskless Array Synthesizer (MAS) by light-directed solid-phase peptide synthesis using an amino-functionalized plastic support (Greiner Bio-One, Kremsmünster, Austria) coupled with a 6-aminohexanoic acid linker and amino acid derivatives carrying a photosensitive 2-(2-nitrophenyl) propyloxycarbonyl (NPPOC) protection group (Orgentis Chemicals, Gatersleben, Germany). To reduce instrument variance, each peptide was printed at 5 locations on the array. Samples were diluted 1:100 in binding buffer $(0.01 \mathrm{M}$ Tris- $\mathrm{Cl}, \mathrm{pH} 7.4$, $1 \%$ alkali-soluble casein, $0.05 \%$ Tween-20). Diluted sample aliquots and negative controls containing binding buffer only were bound to arrays overnight at $4{ }^{\circ} \mathrm{C}$. After binding, the arrays were washed $3 \times$ in wash buffer $(1 \times$ TBS, 0.05\% Tween-20), 10 min per wash. Primary sample binding was detected via 8F1-biotin mouse anti-primate IgG (NIH nonhuman primate reagent resource) secondary antibody. The secondary antibody was diluted 1:10,000 (final concentration $0.1 \mathrm{ng} / \mu \mathrm{L}$ ) in secondary binding buffer $(1 \times$ TBS, $1 \%$ alkali-soluble casein, $0.05 \%$ Tween-20), incubated with arrays for $3 \mathrm{~h}$ at room temperature, and then washed $3 \times$ in wash buffer (10 min per wash) and in reagent-grade water for $30 \mathrm{~s}$. The secondary antibody was labeled with Cy5-Streptavidin (GE Healthcare; $5 \mathrm{ng} / \mu \mathrm{L}$ in $0.5 \times \mathrm{TBS}$, $1 \%$ alkali-soluble casein, $0.05 \%$ Tween-20) for $1 \mathrm{~h}$ at room temperature, and then the array was washed $2 \times$ for $1 \mathrm{~min}$ in $1 \times$ TBS and washed once for $30 \mathrm{~s}$ in reagent-grade water. The fluorescent signal of the secondary antibody was detected by scanning at $635 \mathrm{~nm}$ at $2 \mu \mathrm{m}$ resolution and $25 \%$ gain, using an MS200 microarray scanner (Roche NimbleGen, Madison, WI, USA). The raw fluorescence signal intensity values were $\log 2$ transformed, and the median transformed intensity of the redundant amino acid peptides was determined. Next, background subtraction was performed using the blank samples as background reactivity. Fold change from 0 DPI was calculated by subtracting the respective 0 DPI reactivity on a per amino acid peptide and per animal basis. The peptide array datasets and Jupyter 
notebooks that create the data analysis environment and reproducibly perform the data analyses can be downloaded [24].

\subsection{Flow Cytometry}

Cryopreserved peripheral blood mononuclear cells (PBMCs) were thawed at $37^{\circ} \mathrm{C}$ and washed in R10 media (RPMI containing 10\% fetal bovine serum (FBS)). Between 3-5 million washed cells were transferred to $1.2 \mathrm{~mL}$-cluster tubes and resuspended in phosphate-buffered saline (PBS) supplemented with 10\% FBS containing fluorescence-activated cell sorting (FACS) buffer. Cells were stained at $37^{\circ} \mathrm{C}$ with a mastermix of antibodies against CD38 (clone AT1, FITC conjugate, $20 \mu \mathrm{L}$ ), CD3 (clone SP34-2, Alexa Fluor 700 conjugate, $3 \mu \mathrm{L}$ ), CD8 (clone SK1, Brilliant Violet 510 conjugate, $2.5 \mu \mathrm{L}$ ), CD20 (clone 2H7, Brilliant Violet 650 conjugate, $2 \mu \mathrm{L}$ ), and CD4 (clone L200, Brilliant Violet 711 conjugate, $5 \mu \mathrm{L}$ ) antigens. All antibodies were obtained from BD BioSciences (San Jose, CA, USA), except the CD38-specific antibody, which was purchased from Stemcell Technologies (Vancouver, BC, Canada). Cells were also stained with LIVE/DEAD Fixable Near-IR during this time (Invitrogen, Carlsbad, CA). Cells were washed twice with FACS buffer and fixed with $0.125 \mathrm{~mL}$ of $2 \%$ paraformaldehyde for $20 \mathrm{~min}$. After an additional wash, the cells were permeabilized using Bulk Permeabilization Reagent from Invitrogen (Carlsbad, CA, USA). The cells were stained for 15 min with an antibody against Ki67 (clone B56, Alexa Fluor 647 conjugate, $5 \mu \mathrm{L}$ ) in the presence of permeabilizer. The cells were then washed twice and resuspended in $0.125 \mathrm{~mL}$ of $2 \%$ paraformaldehyde for an additional $20 \mathrm{~min}$. After a final wash and resuspension with $0.125 \mathrm{~mL}$ of FACS buffer, all samples were run on a BD LSRII Flow Cytometer within $24 \mathrm{~h}$. Flow data were analyzed using Flowjo version 9.8.2.

\subsection{Statistical Analysis}

Information on statistical tests used to determine significance can be found in corresponding figure legends. All statistical analyses except those involving sequence analysis and peptide array analysis were performed using Prism7 (GraphPad Software, La Jolla, CA).

\subsection{Data Accessibility}

The sequence of the SWBV-1 inoculum used in this study can be found in GenBank (\#MH686150). SWBV sequencing reads pertaining to this study can be found online at [21] under submission SRP155732 containing the accessions: SAMN09729057, SAMN09729058, SAMN09729059, and SAMN09729060. The workflow used to perform sequence analysis can be found at [24].

\section{Results}

\subsection{Prevalence of SWBV Infection at SNPRC and Selection of SWBV-Nä̈ve Baboons}

To further define the prevalence of SWBV infection in baboons at SNPRC, we screened blood collected from 30 random baboons-19 olive baboons (Papio Anubis); 10 olive-yellow (Papio cynocephalus) hybrid baboons; and 1 hamadryas-olive (Papio hamadryas) hybrid baboon-as well as 6 former olive baboon cage-mates of two olive baboons known to be SWBV-infected using a SWBV-specific qRT-PCR assay described previously. Of these animals, only one olive baboon (a former cage-mate), baboon 15290, had SWBV RNA detectable in its blood at a titer of $3.4 \times 10^{5}$ vRNA copies /mL of plasma, for a prevalence of roughly $3 \%$ (Figure $1 \mathrm{~A}, \mathrm{~B}$ ). Unbiased deep sequencing of this specimen did not result in the identification of any other potential pathogens [20]. To minimize the likelihood of prior SWBV infection, we selected four adult male baboons from the specific-pathogen free (SPF) colony at SNPRC. These animals had tested negative for a variety of common primate pathogens, were brought into the SPF colony as infants, and reared separately from the general colony. These baboons also tested negative for SWBV infection by qRT-PCR. 
A Prevalence of SWBV at SNPRC
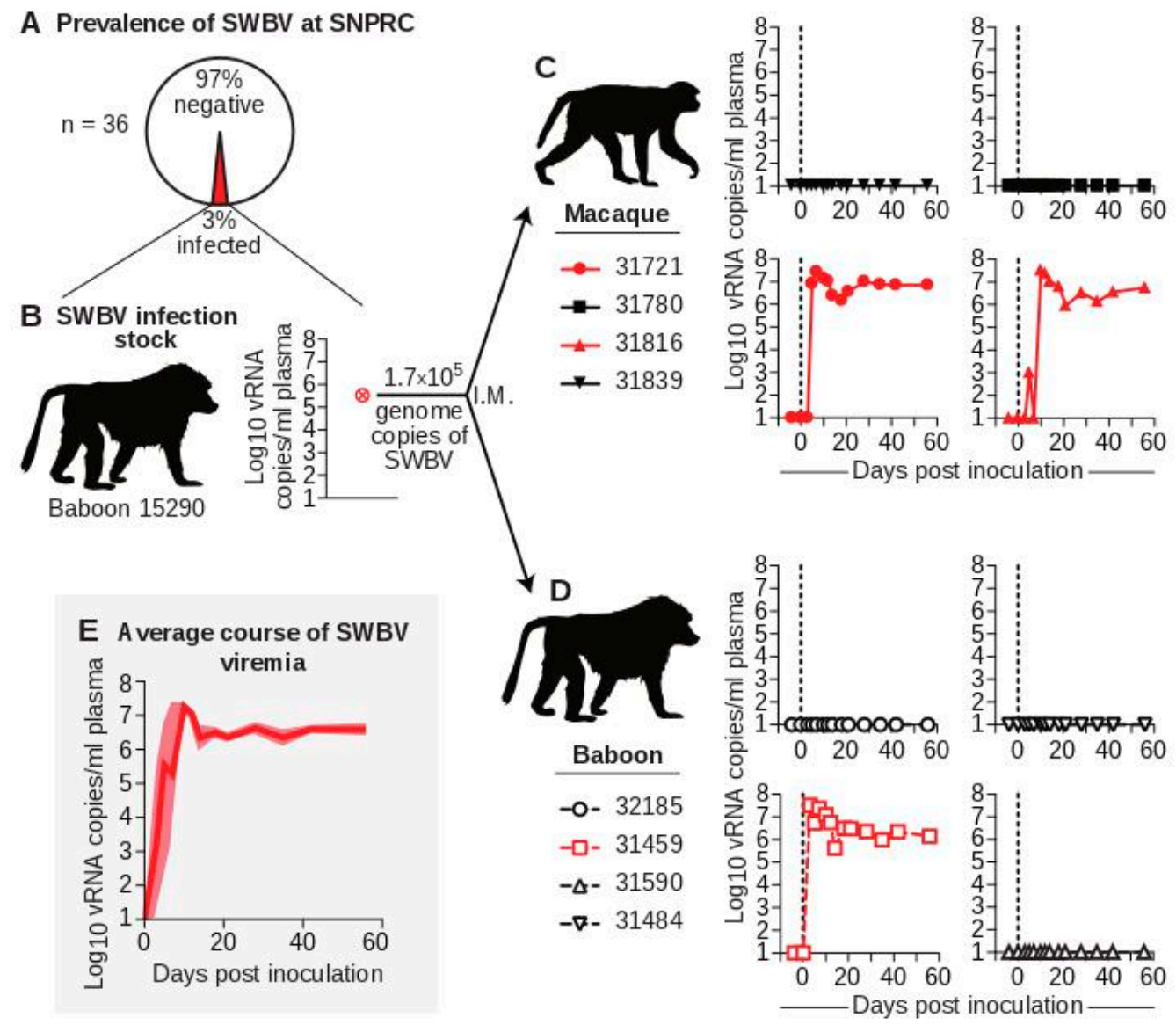

Figure 1. Infection of baboons and macaques with SWBV. (A) Thirty-six baboons from the specific-pathogen free (SPF) colony at SNPRC were screened for SWBV infection using qRT-PCR. (B) This screening identified one SWBV+ baboon. Serum from this animal was used to inoculate (C) macaques (solid symbols with solid lines) and (D) baboons (open symbols with dashed lines), resulting in productive infection (red) or no infection (black) for animals from each species. Note: coloring and symbols denoting species, animal ID, and infection-status are used consistently throughout the manuscript. (E) The average course of SWBV viremia from all infected animals is in dark red, with the standard error of the mean shown in lighter red.

\subsection{The Course of SWBV Infection in Baboons and Macaques}

To study differences in SWBV infection between baboons and macaques, we inoculated four olive baboons (Papio anubis, hereafter referred to as "baboons") and four rhesus monkeys (Macaca mulatta, hereafter referred to as "macaques") intramuscularly with $500 \mu \mathrm{L}$ of serum from baboon 15290 containing $1.7 \times 10^{5}$ vRNA copies of SWBV and quantified viremia using qRT-PCR (Figure 1C,D). Five animals (two macaques and three baboons) had no detectable SWBV viremia at any time point. Three animals (two macaques and one baboon) became productively infected. Among the infected animals, the course of SWBV infection was highly predictable: plasma viremia peaked between $1 \times 10^{7}$ and $1 \times 10^{8}$ vRNA copies/mL at 3-10 DPI, followed by a relative nadir and then establishment of a stable set-point between $1 \times 10^{6}$ and $1 \times 10^{7} \mathrm{vRNA}$ copies $/ \mathrm{mL}$ for the remainder of the study (56 d) (Figure 1E).

\subsection{SWBV Does not Cause Overt Disease in Baboons or Macaques}

We closely monitored all animals for signs of disease using a comprehensive clinical scoring rubric. At no time-point did any animal become clinically ill (score $\geq 5$ ), nor were significant differences between SWBV-infected and uninfected animals noted (Figure 2A). This result is in stark contrast 
to past studies of SHEV or SHFV infection in crab-eating macaques, stump-tailed macaques, and rhesus monkeys, which resulted in extremely high morbidity and mortality (Figure 2B). No significant changes from baseline vital signs, or vital sign differences between SWBV-infected and uninfected animals of either species were observed (Figure 2C).

A Clinical score

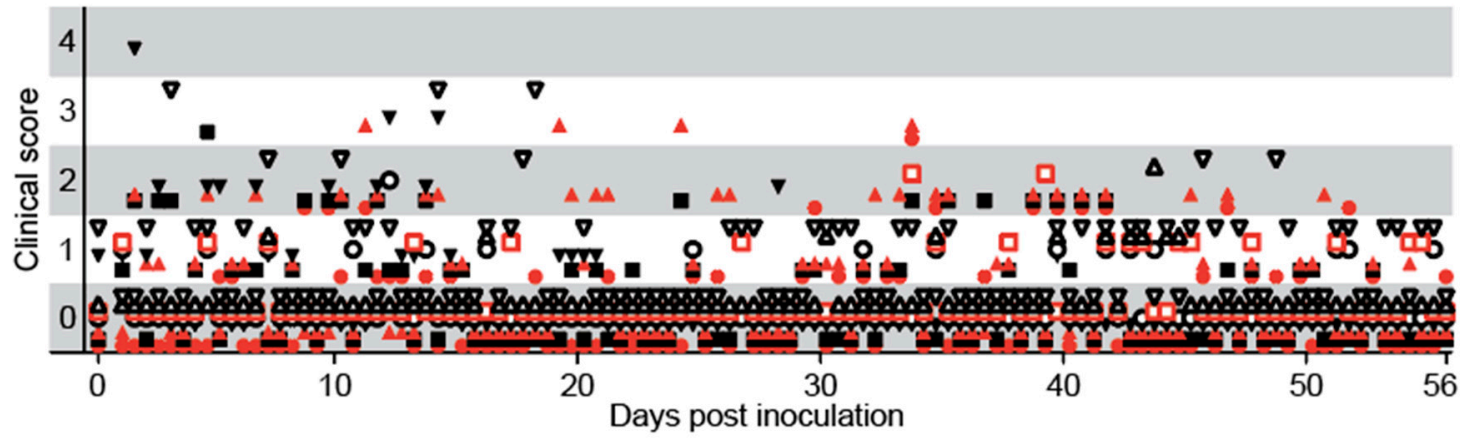

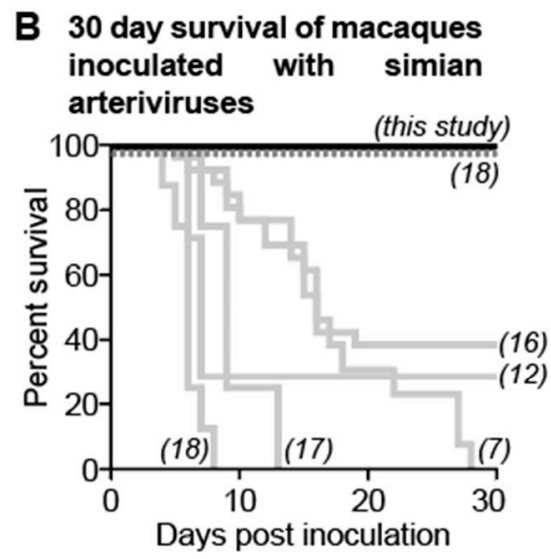

- SWBV (baboon)

.... KRCV-1 (red colobus)

SHFV* (unknown)
C Vital signs
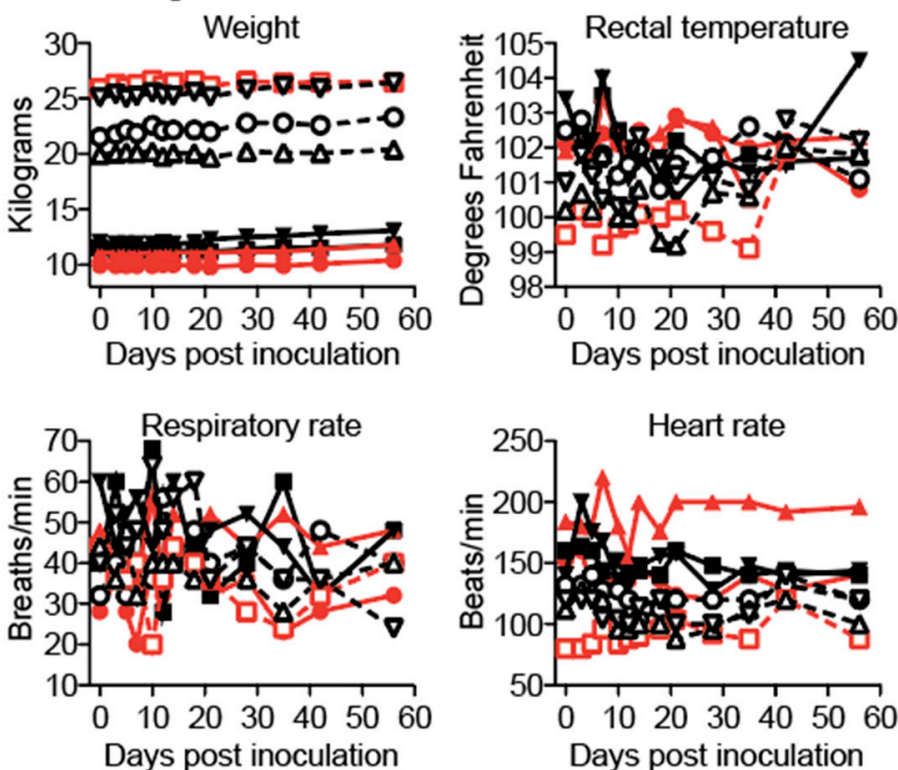

Figure 2. Clinical evaluation of baboons and macaques inoculated with SWBV. (A) Animals were evaluated for signs of disease twice per day using a comprehensive clinical scoring rubric in which a score of $<5$ was deemed within normal limits. (B) For macaques, mortality was also assessed (black line) and compared to previous studies of simarterivirus infection in macaques (corresponding references in italics); the virus and hosts for the simarteriviruses shown are found below the graph in parentheses (SHFV has an asterisk because the natural host is not known and the sequence identity of the viruses used in some of these studies has never been confirmed). Vital signs for baboons and macaques are shown in (C). Macaques are shown as solid symbols with solid lines, baboons are shown as open symbols with dashed lines; infected animals are shown in red and uninfected animals are shown in black.

To examine more sensitive markers of infection, inflammation, and organ dysfunction, we performed a battery of laboratory tests on each animal over the course of the study, the most pertinent of which are shown (Figure 3). No significant changes in white blood cell counts or the numbers of leukocyte subsets were seen over the course of the experiment for either SWBV-infected or uninfected animals (Figure 3A). Additionally, in stark contrast to previous studies of SHFV in macaques, no coagulation abnormalities were observed for any of the animals, as measured by blood 
concentrations of fibrinogen or functional clotting assays, including pro-thrombin time (PT) and partial thromboplastin time (PTT) (Figure 3B). Blood concentrations of creatinine, aspartate aminotransferase, and alanine aminotransferase-which serve as markers of kidney function and liver cell death, respectively-were not significantly different between SWBV-infected and uninfected animals (Figure 3C). Finally, necropsies performed at 56 DPI revealed no gross or microscopic differences between SWBV-infected and uninfected animals.

\section{A Hematology}
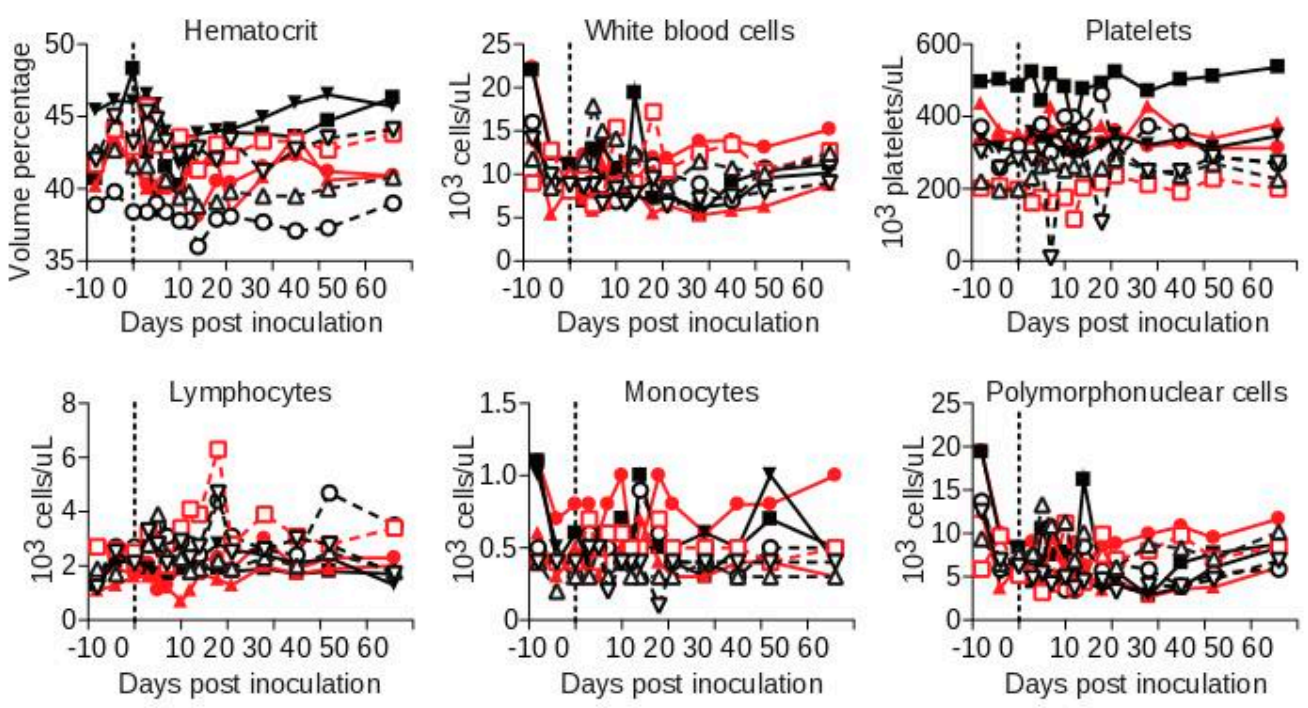

B Coagulation
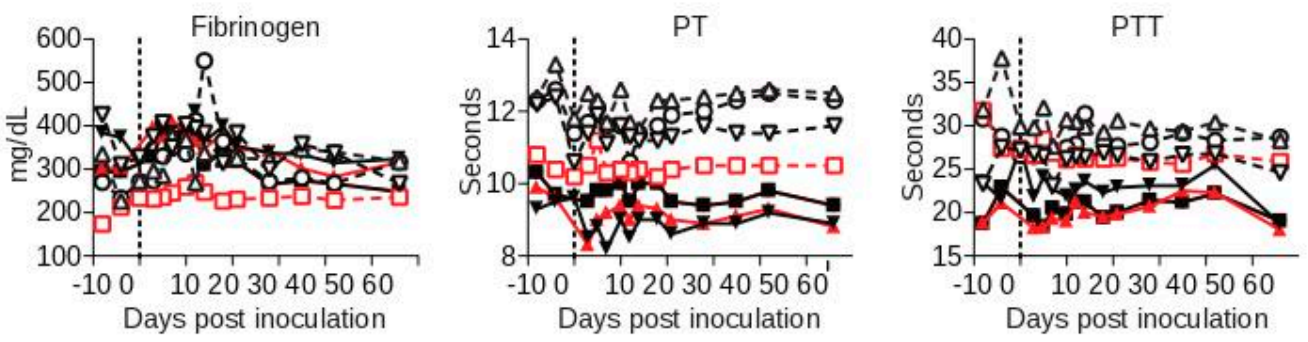

C End-organ damage
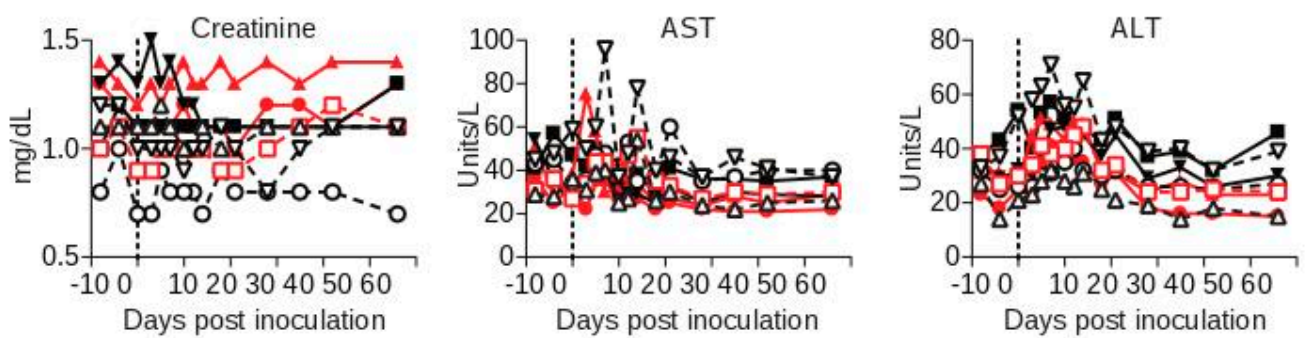

Figure 3. Laboratory evaluation of baboons and macaques inoculated with SWBV. Clinical chemistry, hematology, and coagulation studies were performed on fresh blood at the SNPRC clinical laboratory. Macaques are shown as solid symbols with solid lines, baboons are shown as open symbols with dashed lines; infected animals are shown in red and uninfected animals are shown in black.

\subsection{Arterivirus-Specific Adaptive Immune Responses in SWBV-Infected Macaques and Baboons}

To evaluate immune responses to SWBV, we assessed immune-cell activation in peripheral blood mononuclear cells (PBMC) from SWBV-inoculated baboons and macaques using flow cytometry 
(Figure 4A). No significant deviations from baseline levels of $\mathrm{CD}^{+} \mathrm{T}$ cell, $\mathrm{CD} 8^{+} \mathrm{T}$ cell, or natural killer (NK) cell activation were observed in uninfected animals (Figure 4B). However, the percentage of activated CD4+ T cells, CD8+ T cells, and NK cells increased substantially in animals that became SWBV-viremic (Figure 4B): In macaque 31721 and baboon 31459, the percentage of activated immune cells peaked at 10 DPI and then quickly returned to near-baseline levels; activation of these immune-cell subsets peaked later in macaque 31816 (between d 14 and 21) with the proportion of activated CD8+ T cells remaining elevated through 56 DPI.

\section{A Flow cytometry gating strategy}

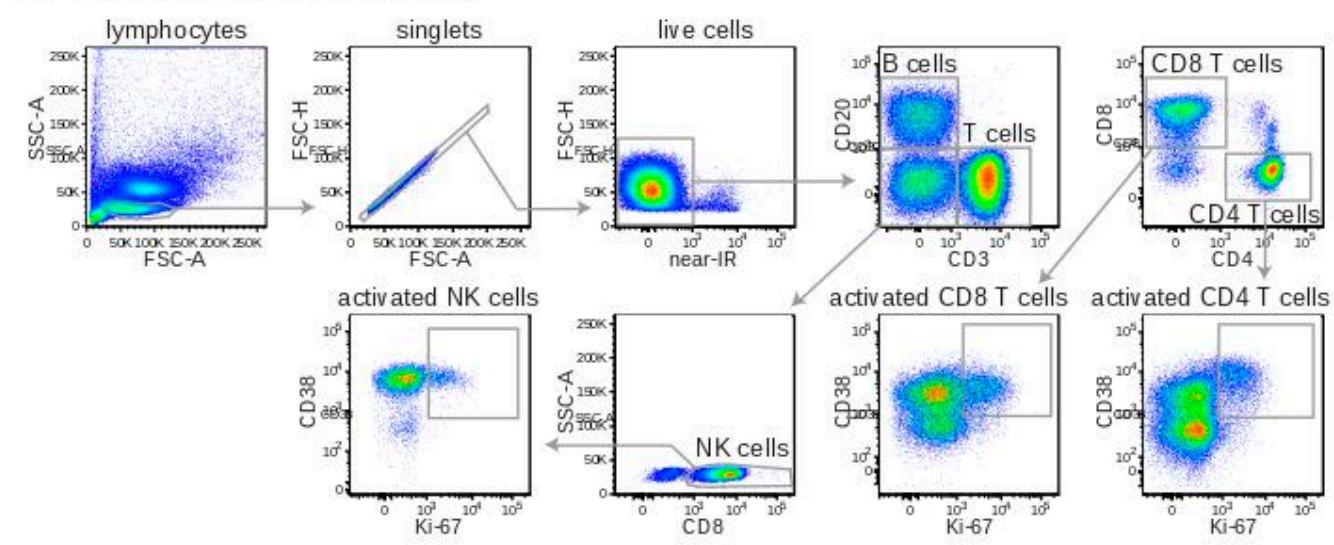

\section{B Activation of lymphocyte subsets}
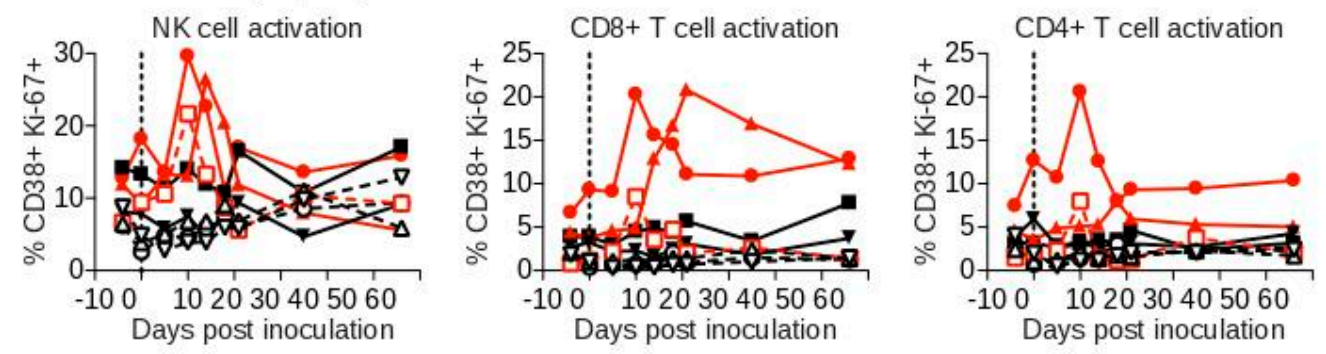

Figure 4. Flow cytometric analysis of peripheral blood lymphocytes from baboons and macaques inoculated with SWBV. Flow cytometry was performed on peripheral blood mononuclear cells (PBMC) that were purified and cryopreserved at the time of collection. Lymphocytes were phenotyped according to cell-surface markers, and co-expression of CD38 and Ki-67 was used as a marker of cellular activation. (A) Shows a representative sample at a single time-point with the gating strategy; (B) shows flow cytometry data from each animal over the course of the experiment. Macaques are shown as solid symbols with solid lines, baboons are shown as open symbols with dashed lines; infected animals are shown in red and uninfected animals are shown in black.

We also assessed antibody responses to SWBV using a microarray of 16-mer peptides that spanned the SWBV proteome with a one amino acid offset. Many arterivirus envelope glycoproteins are predicted to have relatively unstructured proteins and have been shown to elicit antibodies that recognize linear epitopes, suggesting that many of the antibodies identified by this approach would be functional [25]. No SWBV-specific antibodies were detected at levels above background in any animals that were aviremic throughout the study (Figure 5A). In SWBV-infected animals, SWBV-specific antibodies became detectable between 12 and 28 DPI. Although multiple envelope glycoproteins were targeted by antibodies in each of the SWBV-infected animals, common responses were focused against glycoproteins 5 and 6 (GP5 and GP6), with the most robust responses generated to GP5 (Figure 5B). In particular, two distinct regions/epitopes of GP5, corresponding to amino acids 28-62 and 76-111, were targeted, with the macaques mounting more robust responses to $\mathrm{GP}_{28-62}$ and the baboon mounting a more robust response to $\mathrm{GP}_{76-111}$. Multiple attempts to propagate SWBV on cultured 
primary and immortalized cells were unsuccessful, preventing further functional characterization of these antibody responses.

A Antibody response to SWBV envelope glycoproteins over the course of infection

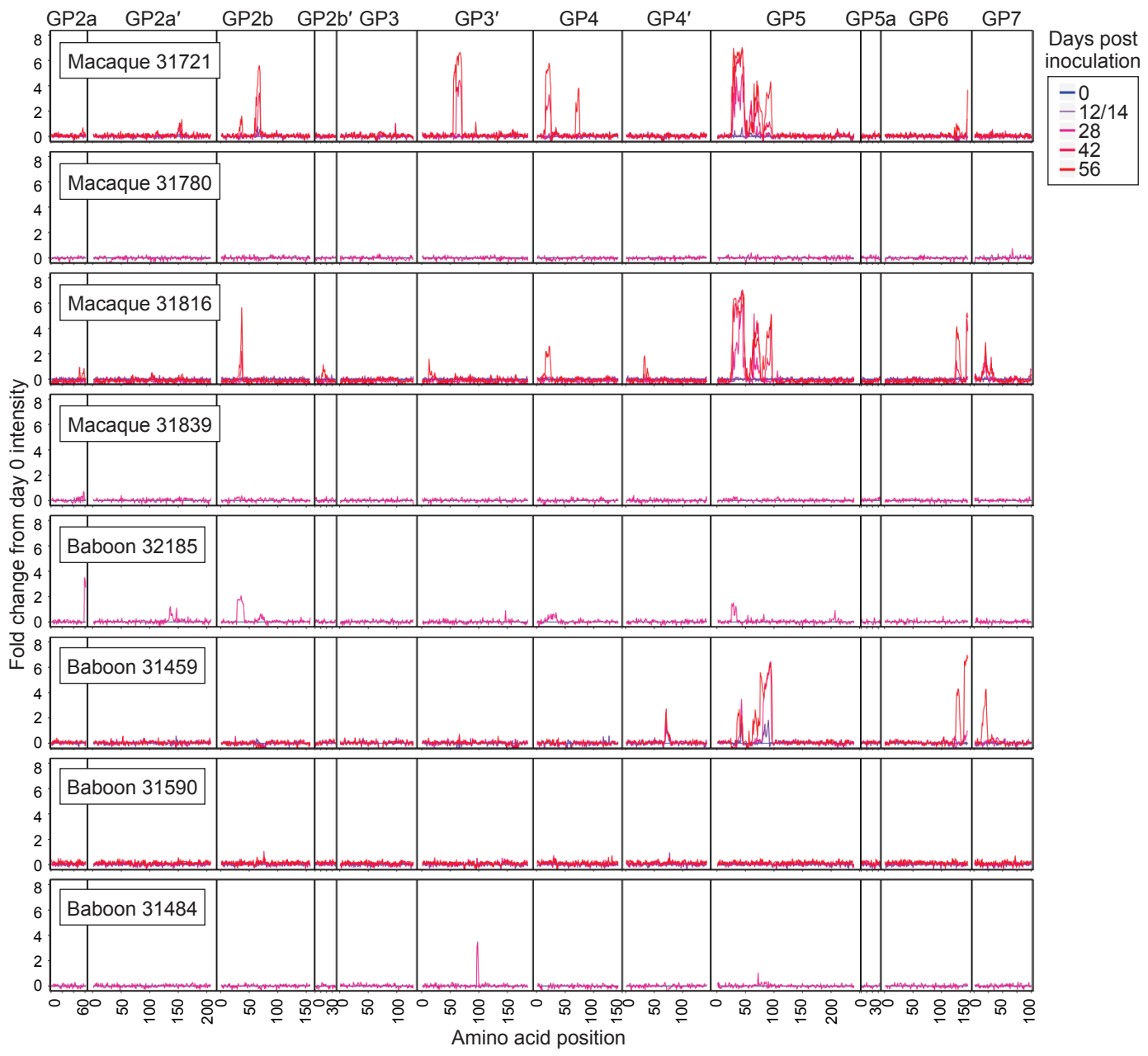

B Antibody response to SWBV GP5 over the course of infection
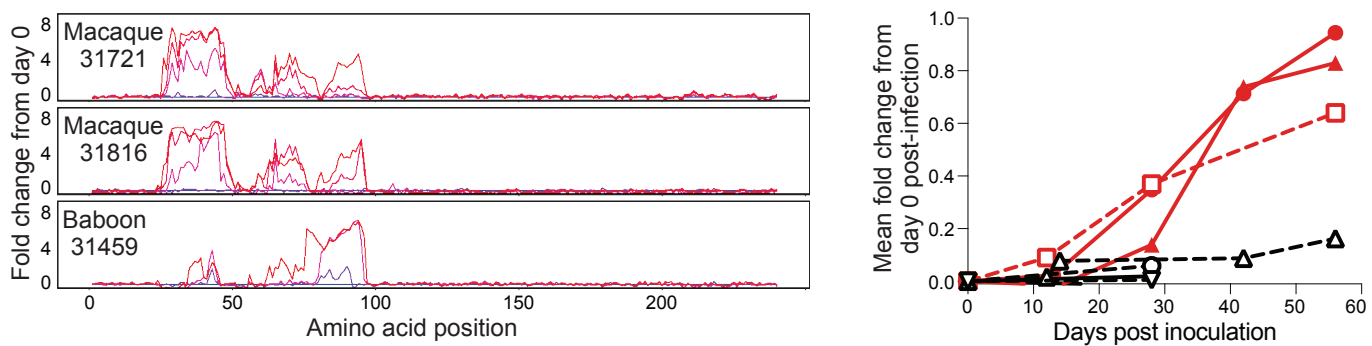

Figure 5. Peptide array analysis of antibodies generated by baboons and macaques inoculated with SWBV. A custom-designed array of 16-mer peptides spanning each of the SWBV proteins predicted from the SWBV inoculum nucleotide sequence was constructed, and plasma from SWBV-infected animals was overlaid on this array to identify SWBV-specific antibodies. (A) Intensity of antibodies binding to peptides corresponding to the envelope glycoproteins of SWBV, normalized to the day 0 post-inoculation intensity for each peptide from each animal, with day 0 shown in blue and subsequent days shown in increasing shades of red. Note, numbers on the $\mathrm{X}$-axis correspond to the first amino acid in the 16-mer peptide. (B) Normalized intensities over time from the GP5 protein of the three animals that became infected with SWBV; the graph to the right shows the mean fold change from day 0 post-inoculation for all GP5 peptides, with SWBV-infected animals shown in red. 


\subsection{Intra-Host Evolution of SWBV over the Course of Infection}

We examined the genetic changes in SWBV that accumulated over the course of each animal's infection by performing deep sequencing on plasma collected from each viremic animal. Compared to the virus used for inoculation, we found mutations in nearly every SWBV gene (Table S1), many of which were not present at detectable levels in the inoculum. However, the density of non-synonymous mutations was not evenly distributed, with many mutations occurring in the genes encoding envelope glycoproteins, particularly the GP5-encoding ORF5 gene.

\section{Discussion}

In this study, we attempted to infect baboons and macaques with a baboon arterivirus, SWBV, to more fully characterize the pathogenesis of simarterivirus infection in natural versus non-natural hosts. Given the history of simarteriviruses causing severe disease in macaques, we expected macaques infected with SWBV to develop significant disease. Surprisingly, however, SWBV resulted in clinically unapparent disease in macaques, despite high-titer and persistent viremia. While high-titer and persistent viremia is likely the most common course of simarterivirus infection in naturally infected African monkeys [5], this finding is (to our knowledge) the first example of persistent simarterivirus infection in macaques. The lack of disease observed in this study is in contrast to the cotemporaneous study performed by Cornish et al., in which patas monkeys and rhesus monkeys both developed laboratory aberrations and physical signs of disease following infection with SHFV. Therefore, simarterivirus infections appears to be: 1) sub-clinical in African monkeys [6,20,26-29], 2) pathogenic in African monkeys [30-32] and Cornish et al., 3) sub-clinical in Asian-origin macaques (this study), and 4) pathogenic in Asian-origin macaques [7-18]. The viral factors that that render some simarteriviruses (e.g., SWBV) non-pathogenic but confer virulence to other simarteriviruses (e.g., SHFV) remain to be defined, but clearly cannot be explained by host differences alone.

Analysis of immune responses to SWBV and SHFV provided no additional explanation for the range of disease observed in this study and the study by Cornish et al. In this study, SWBV infection persisted in all three animals that became infected despite SWBV-specific immune responses. Antibody responses to SWBV primarily were directed against the major envelope glycoprotein, GP5. More specifically, antibodies were raised against two N-terminal epitopes in GP5 corresponding to amino acids 28-62 and 76-111. Although the relative strength of these responses differed between the macaques and the baboon, with antibodies to the epitope corresponding to amino acids 28-62 dominating in the macaques and antibodies to the epitope corresponding to amino acids 76-111 dominating in the baboon, none of these responses were capable of achieving viral clearance. Antibody responses to the other arteriviruses have also been mapped to two N-terminal epitopes in GP5 [33-36]. Intriguingly, the more N-terminal of these two epitopes in porcine reproductive and respiratory syndrome virus (PRRSV) is immunodominant but elicits a non-neutralizing response, referred to by some as a "decoy epitope" [37]. A similar mechanism of immune evasion may be employed by simarteriviruses. Indeed, we identified amino acid changes in these epitopes-including mutations in predicted glycosylation sites- that would presumably abrogate antibody binding and allow SWBV to establish persistent infection by escaping antibody responses. Interestingly, the vast majority of non-synonymous mutations in the viruses from these animals are found in the sequence corresponding to the more C-terminal epitope (GP5 amino acids 76-111), further suggesting that antibodies to the more N-terminal epitope (GP5 amino acids 28-62) are non-neutralizing and do not exert a significant selective pressure on the virus (Figure 6). This also suggests that antibodies to the C-terminal epitope exert a stronger selection pressure on the virus, potentially implicating this epitope as a target of neutralizing antibodies, similar to what has been observed in other arteriviruses. 


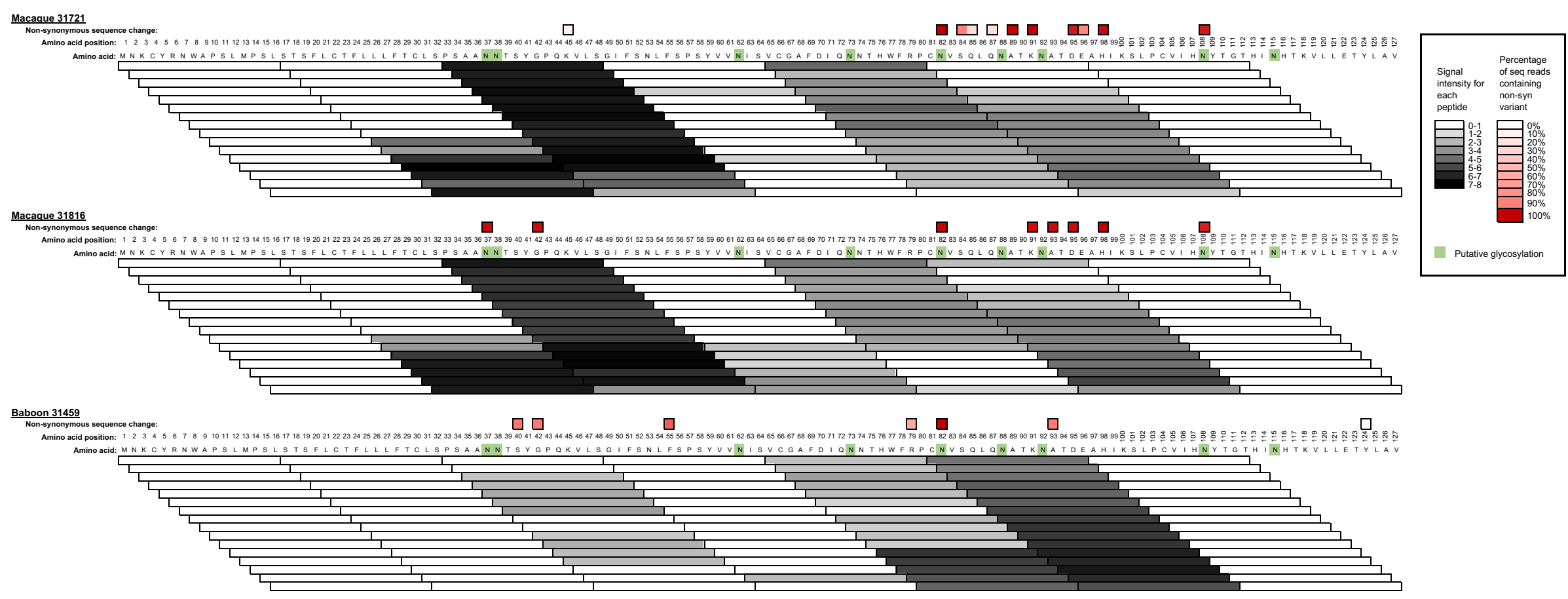

Figure 6. Correlation of SWBV mutations with antibody responses. Antibody binding intensity data (see Figure 5) are shown for each peptide covering the region of interest in GP5 as a heat-mapped scale of 0 (white) to $>7$ (black). Overlaid are the non-synonymous sequencing changes corresponding to each amino acid of GP5 (see Table S1), with a heat-map highlighting variants contained in 20\% (pink) to 100\% (dark red) of deep sequencing reads. Amino acids in green highlight residues with predicted N-linked glycosylation. Data for each animal correspond to analysis of the day 56 post-inoculation time-point with the exception of sequencing data from baboon 31459, for which day 12 post-inoculation. 
The majority of animals in this study (5/8) did not become infected upon inoculation with SWBV. This finding seems unlikely to be due to pre-existing immunity from a prior SWBV infection. Pre-existing immunity would have presumably resulted in detectable anti-SWBV antibodies and/or robust proliferation of immune cells following SWBV inoculation-neither of which were seen. Immunity due to prior or ongoing infection with a different simarterivirus is also unlikely, as heterologous protection between divergent simarterivirus strains is not seen [18]. A technical error in the inoculation process is also possible but unlikely, considering that animals in each group were successfully infected. SWBV also replicated to high titers in animals that became viremic, arguing against the potential that SWBV was poorly adapted to replication in the baboon or macaque host. Therefore, it is possible that the uninfected animals were innately resistant to simarterivirus infection, or that we used a dose of SWBV $\left(1.7 \times 10^{5}\right.$ vRNA copies $)$ that only infects $50 \%$ of animals ( 1 infectious dose $50 \%\left(\mathrm{ID}_{50}\right)$ ).

Several parallels can be drawn between the simarteriviruses and simian immunodeficiency viruses (SIVs): both naturally infect monkeys throughout sub-Saharan Africa and have variable propensities for causing disease upon transmission to non-natural primate hosts. Cross-species transmission of SIVs to humans and other great apes has had devastating consequences (e.g., the global HIV-1-pandemic), but not all SIVs have the same potential for infecting and causing disease in humans. By analogy, the consequences of simarterivirus infection in humans remains unknown, and different simarteriviruses may have different propensities for infecting and causing disease in humans [5]. If macaques are the best proxy for assessing such variables (as they are in the case of $\mathrm{SIV} / \mathrm{HIV}$ ), then it is possible that simarteriviruses are capable of causing a range of disease in humans, including asymptomatic infection. Consequently, we believe that simarterivirus research should be intensified to allow an appropriate risk assessment.

Supplementary Materials: The following are available online at http:/ / www.mdpi.com/1999-4915/10/12/701/ s1, Figure S1: Flow cytometry gating strategy; Table S1: Single nucleotide variants found at $>5 \%$ in SWBV from infected animals; Table S2: Primers used for amplicon sequencing of SWBV.

Author Contributions: Conceptualization, A.L.B., J.H.K, and D.H.O.; methodology, A.L.B and C.B.; software, D.A.B.; formal analysis, A.L.B, C.B., M.S., and D.A.B.; investigation, A.L.B, C.B., M.S., C.N., D.C., B.G., K.B., and D.A.B.; writing—original draft preparation, A.L.B and C.B.; writing—review and editing, J.H.K, R.F.J., J.P.C., D.H.O., and R.L.

Funding: This investigation was supported by a Pilot Grant from the Southwest National Primate Research Center grant P51OD011133 from the Office of Research Infrastructure Programs (ORIP), National Institutes of Health. This work was also made possible by funding to the Wisconsin National Primate Research Center (P51RR000167) from ORIP. This research was conducted in part at a facility constructed with support from Research Facilities Improvement Program grant numbers RR15459-01 and RR020141-01. A.L.B. performed this work with support from the University of Wisconsin-Madison's Medical Scientist Training Program (MSTP) (grant T32GM008692) and the Physician Scientist Training Program (PSTP) sponsored by the Department of Pathology and Immunology at Washington University in St. Louis School of Medicine. The authors thank the University of Wisconsin, Department of Pathology and Laboratory Medicine and the WNPRC for funding and the use of its facilities and services. This work was also funded in part through the National Institute of Allergy and Infectious Diseases (NIAID) Division of Intramural Research (J.P.C. and R.F.J.) and the Battelle Memorial Institute's prime contract with the US NIAID under Contract No. HHSN272200700016I (J.H.K.). This publication's contents are solely the responsibility of the author(s) and do not necessarily represent the official views of ORIP, of ORIP, the US National Institutes of Health, NIAID, US Department of Health and Human Services, or of the institutions and companies affiliated with the authors. The funders of this research had no role in study design, data collection and analysis, decision to publish, or preparation of the manuscript. All authors edited and approved the manuscript.

Conflicts of Interest: The authors declare no conflict of interest.

\section{References}

1. Davies, T.J.; Pedersen, A.B. Phylogeny and geography predict pathogen community similarity in wild primates and humans. Proc. Biol. Sci. R. Soc. 2008, 275, 1695-1701. [CrossRef] [PubMed]

2. Cooper, N.; Nunn, C.L. Identifying future zoonotic disease threats: Where are the gaps in our understanding of primate infectious diseases? Evol. Med. Public Health 2013, 2013, 27-36. [CrossRef] [PubMed] 
3. Calvignac Spencer, S.; Leendertz, S.A.J.; Gillespie, T.R.; Leendertz, F.H. Wild great apes as sentinels and sources of infectious disease. Clin. Microbiol. Infect. 2012, 18, 521-527. [CrossRef] [PubMed]

4. Hoppe, E.; Pauly, M.; Gillespie, T.R.; Akoua-Koffi, C.; Hohmann, G.; Fruth, B.; Karhemere, S.; Madinda, N.F.; Mugisha, L.; Muyembe, J.-J. Multiple cross-species transmission events of human adenoviruses (HAdV) during hominine evolution. Mol. Biol. Evol. 2015, 8, 2072-2084. [CrossRef] [PubMed]

5. Bailey, A.L.; Lauck, M.; Sibley, S.D.; Friedrich, T.C.; Kuhn, J.H.; Freimer, N.B.; Jasinska, A.J.; Phillips-Conroy, J.E.; Jolly, C.J.; Marx, P.A.; et al. Zoonotic Potential of Simian Arteriviruses. J. Virol. 2015, 90, 630-635. [CrossRef] [PubMed]

6. Bailey, A.L.; Lauck, M.; Ghai, R.R.; Nelson, C.W.; Heimbruch, K.; Hughes, A.L.; Goldberg, T.L.; Kuhn, J.H.; Jasinska, A.J.; Freimer, N.B. Arteriviruses, pegiviruses, and lentiviruses are common among wild African monkeys. J. Virol. 2016, 15, 6724-6737. [CrossRef] [PubMed]

7. Palmer, A.E.; Allen, A.M.; Tauraso, N.M.; Shelokov, A. Simian hemorrhagic fever. I. Clinical and epizootiologic aspects of an outbreak among quarantined monkeys. Am. J. Trop. Med. Hyg. 1968, 17, 404-412. [CrossRef] [PubMed]

8. Allen, A.M.; Palmer, A.E.; Tauraso, N.M.; Shelokov, A. Simian hemorrhagic fever. II. Studies in pathology. Am. J. Trop. Med. Hyg. 1968, 17, 413-421. [CrossRef]

9. Tauraso, N.M.; Shelokov, A.; Palmer, A.E.; Allen, A.M. Simian hemorrhagic fever. III. Isolation and characterization of a viral agent. Am. J. Trop. Med. Hyg. 1968, 17, 422-431. [CrossRef]

10. Lapin, B.A.; Pekerman, S.M.; Iakovleva, L.A.; Dzhikidze, E.K.; Shevtsova, Z.V.; Kuksova, M.I.; Dan'ko, L.V.; Krylova, R.I.; Akbroit, E.I.; Agrba, V.Z. Hemorrhagic fever in monkeys. Vopr. Virusol. 1967, 12, 168-173.

11. Espana, C. Review of some outbreaks of viral disease in captive nonhuman primates. Lab. Anim. Sci. 1971, 21, 1023-1031. [PubMed]

12. Abildgaard, C.; Harrison, J.; Espana, C.; Spangler, W.; Gribble, D. Simian hemorrhagic fever: Studies of coagulation and pathology. Am. J. Trop. Med. Hyg. 1975, 24, 537-544. [CrossRef] [PubMed]

13. London, W.T. Epizootiology, transmission and approach to prevention of fatal simian haemorrhagic fever in rhesus monkeys. Nature 1977, 268, 344-345. [CrossRef] [PubMed]

14. Renquist, D. Outbreak of simian hemorrhagic fever. J. Med. Primatol. 1990, 19, 77-79. [PubMed]

15. Dalgard, D.W.; Hardy, R.J.; Pearson, S.L.; Pucak, G.J.; Quander, R.V.; Zack, P.M.; Peters, C.J.; Jahrling, P.B. Combined simian hemorrhagic fever and Ebola virus infection in cynomolgus monkeys. Lab. Anim. Sci. 1992, 42, 152-157. [PubMed]

16. Johnson, R.F.; Dodd, L.E.; Yellayi, S.; Gu, W.; Cann, J.A.; Jett, C.; Bernbaum, J.G.; Ragland, D.R.; St Claire, M.; Byrum, R.; et al. Simian hemorrhagic fever virus infection of rhesus macaques as a model of viral hemorrhagic fever: Clinical characterization and risk factors for severe disease. Virology 2011, 421, 129-140. [CrossRef]

17. Vatter, H.A.; Donaldson, E.F.; Huynh, J.; Rawlings, S.; Manoharan, M.; Legasse, A.; Planer, S.; Dickerson, M.F.; Lewis, A.D.; Colgin, L.M.; et al. A simian hemorrhagic fever virus isolate from persistently infected baboons efficiently induces hemorrhagic fever disease in Japanese macaques. Virology 2015, 474, 186-198. [CrossRef]

18. Wahl-Jensen, V.; Johnson, J.C.; Lauck, M.; Weinfurter, J.T.; Moncla, L.H.; Weiler, A.M.; Charlier, O.; Rojas, O.; Byrum, R.; Ragland, D.R.; et al. Divergent Simian Arteriviruses Cause Simian Hemorrhagic Fever of Differing Severities in Macaques. MBio 2016, 7, e02009-15. [CrossRef]

19. Lauck, M.; Alkhovsky, S.V.; Bào, Y.M.; Bailey, A.L.; Shevtsova, Z.V.; Shchetinin, A.M.; Vishnevskaya, T.V.; Lackemeyer, M.G.; Postnikova, E.; Mazur, S.; et al. Historical Outbreaks of Simian Hemorrhagic Fever in Captive Macaques Were Caused by Distinct Arteriviruses. J. Virol. 2015, 15, 8082-8087. [CrossRef]

20. Bailey, A.L.; Lauck, M.; Sibley, S.D.; Pecotte, J.; Rice, K.; Weny, G.; Tumukunde, A.; Hyeroba, D.; Greene, J.; Correll, M.; et al. Two novel simian arteriviruses in captive and wild baboons (Papio spp.). J. Virol. 2014, 88, 13231-13239. [CrossRef]

21. BioProject. SWBV Sequencing from Macaques and Baboons at SNPRC. Accession number: PRJNA483240. Available online: https: / www.ncbi.nlm.nih.gov/bioproject/483240 (accessed on 7 December 2018).

22. Joint Genome Institute. BBMap Guide. Available online: https://jgi.doe.gov/data-and-tools/bbtools/bbtools-user-guide/bbmap-guide/ (accessed on 7 December 2018).

23. SnpEff Genomic Variant Annotations and Functional Effect Prediction Toolbox. Available online: http: / / snpeff.sourceforge.net/ (accessed on 7 December 2018). 
24. University of Wisconsin-Madison AIDS Vaccine Research Laboratory. Non-pathogenic Infection of Macaques and Baboons with a Baboon Arterivirus. Available online: https:/ / dholk.primate.wisc.edu/project/dho/ public/manuscripts / 201808-SNPRC-SWBV/begin.view? (accessed on 7 December 2018).

25. Dokland, T. The structural biology of PRRSV. Virus Res. 2010, 154, 86-97. [CrossRef] [PubMed]

26. Gravell, M.; Palmer, A.E.; Rodriguez, M.; London, W.T.; Hamilton, R.S. Method to detect asymptomatic carriers of simian hemorrhagic fever virus. Lab. Anim. Sci. 1980, 30, 988-991. [PubMed]

27. Bailey, A.L.; Lauck, M.; Weiler, A.; Sibley, S.D.; Dinis, J.M.; Bergman, Z.; Nelson, C.W.; Correll, M.; Gleicher, M.; Hyeroba, D.; et al. High genetic diversity and adaptive potential of two simian hemorrhagic fever viruses in a wild primate population. PLoS ONE 2014, 9, e90714. [CrossRef] [PubMed]

28. Lauck, M.; Switzer, W.M.; Sibley, S.D.; Hyeroba, D.; Tumukunde, A.; Weny, G.; Taylor, B.; Shankar, A.; Ting, N.; Chapman, C.A.; et al. Discovery and full genome characterization of two highly divergent simian immunodeficiency viruses infecting black-and-white colobus monkeys (Colobus guereza) in Kibale National Park, Uganda. Retrovirology 2013, 10, 107. [CrossRef] [PubMed]

29. Lauck, M.; Sibley, S.D.; Hyeroba, D.; Tumukunde, A.; Weny, G.; Chapman, C.A.; Ting, N.; Switzer, W.M.; Kuhn, J.H.; Friedrich, T.C.; et al. Exceptional simian hemorrhagic Fever virus diversity in a wild african primate community. J. Virol. 2013, 87, 688-691. [CrossRef] [PubMed]

30. Gravell, M.; London, W.T.; Rodriguez, M.; Palmer, A.E.; Hamilton, R.S.; Curfman, B.L. Studies on simian hemorrhagic fever virus infection of patas monkeys - I. Serology. In The Comparative Pathology of Zoo Animals (the Symposia of the National Zoological Park); Montali, R.J., Migaki, G., Eds.; Smithsonian Institution Press: Washington, DC, USA, 1980; pp. 167-170.

31. Gravell, M.; London, W.T.; Leon, M.E.; Palmer, A.E.; Hamilton, R.S. Differences among isolates of simian hemorrhagic fever (SHF) virus. Proc. Soc. Exp. Biol. Med. (N. Y.) 1986, 181, 112-119. [CrossRef]

32. Gravell, M.; London, W.T.; Leon, M.; Palmer, A.E.; Hamilton, R.S. Elimination of persistent simian hemorrhagic fever (SHF) virus infection in patas monkeys. Proc. Soc. Exp. Biol. Med. (N. Y.) 1986, 181, 219-225. [CrossRef]

33. Balasuriya, U.B.; Patton, J.F.; Rossitto, P.V.; Timoney, P.J.; McCollum, W.H.; MacLachlan, N.J. Neutralization determinants of laboratory strains and field isolates of equine arteritis virus: Identification of four neutralization sites in the amino-terminal ectodomain of the $G(L)$ envelope glycoprotein. Virology 1997, 232, 114-128. [CrossRef]

34. Plagemann, P.G. Complexity of the single linear neutralization epitope of the mouse arterivirus lactate dehydrogenase-elevating virus. Virology 2001, 290, 11-20. [CrossRef]

35. Plagemann, P.G.W.; Rowland, R.R.R.; Faaberg, K.S. The primary neutralization epitope of porcine respiratory and reproductive syndrome virus strain VR-2332 is located in the middle of the GP5 ectodomain. Arch. Virol. 2002, 147, 2327-2347. [CrossRef]

36. Plagemann, P.G.W. The primary GP5 neutralization epitope of North American isolates of porcine reproductive and respiratory syndrome virus. Vet. Immunol. Immunopathol. 2004, 102, 263-275. [CrossRef] [PubMed]

37. Ostrowski, M.; Galeota, J.A.; Jar, A.M.; Platt, K.B.; Osorio, F.A.; Lopez, O.J. Identification of neutralizing and nonneutralizing epitopes in the porcine reproductive and respiratory syndrome virus GP5 ectodomain. J. Virol. 2002, 76, 4241-4250. [CrossRef] [PubMed]

(C) 2018 by the authors. Licensee MDPI, Basel, Switzerland. This article is an open access article distributed under the terms and conditions of the Creative Commons Attribution (CC BY) license (http://creativecommons.org/licenses/by/4.0/). 